\title{
MINIMUM COVERING SEIDEL ENERGY OF A GRAPH
}

\author{
M. R. Rajesh Kanna ${ }^{1}$, R. Jagadeesh ${ }^{2}$, Mohammad Reza Farahani ${ }^{3}$ \\ ${ }^{1}$ Post Graduate Department of Mathematics, \\ Maharani's Science College for Women, \\ J. L. B. Road, Mysore - 570 005, India \\ mr.rajeshkanna@gmail.com \\ ${ }^{2}$ Research Scholar, \\ Research and Development Centre, \\ Bharathiar University, Coimbatore 641 046, India. \\ jagadeeshr1978@gmail.com \\ ${ }^{3}$ Department of Applied Mathematics, \\ Iran University of Science and Technology (IUST) Narmak, \\ Tehran, 16844, Iran. \\ mrfarahani88@gmail.com
}

\begin{abstract}
In this paper we have computed minimum covering Seidel energies of a star graph, complete graph, crown graph, complete bipartite graph and cocktail party graphs. Upper and lower bounds for minimum covering Seidel energies of graphs are also established.

Key words and Phrases: Minimum covering set, Minimum covering Seidel matrix, Minimum covering Seidel eigenvalues, Minimum covering Seidel energy of a graph.
\end{abstract}

\begin{abstract}
Abstrak. Dalam paper ini kami menghitung energi Seidel selimut minimum dari graf bintang, graf lengkap, graf mahkota, graf bipartit lengkap dan graf cocktail party. Kami juga memperlihatkan batas atas dan bawah untuk energi Seidel selimut minimum dari suatu graf.

Kata kunci: Himpunan selimut minimum, Matriks Seidel selimut minimum, Nilai eigen Seidel selimut minimum, Energi Seidel selimut minimum dari graf.
\end{abstract}

\section{INTRODUCTION}

The concept of energy of a graph was introduced by I. Gutman [7] in the year 1978. Let $G$ be a graph with $n$ vertices and $m$ edges and let $A=\left(a_{i j}\right)$ be the adjacency matrix of the graph. The eigenvalues $\lambda_{1}, \lambda_{2}, \cdots, \lambda_{n}$ of $\mathrm{A}$, assumed in

2000 Mathematics Subject Classification: Primary 05C50, 05C69.

Received: 27-12-2015, revised: 20-04-2016, accepted: 21-04-2016. 
non increasing order, are the eigenvalues of the graph $G$. As A is real symmetric, the eigenvalues of $G$ are real with sum equal to zero. The energy $E(G)$ of $G$ is defined to be the sum of the absolute values of the eigenvalues of $G$. i.e., $E(G)=$ $\sum_{i=1}^{n}\left|\lambda_{i}\right|$.

For details on the mathematical aspects of the theory of graph energy see the reviews [8], papers $[4,5,9]$ and the references cited there in. The basic properties including various upper and lower bounds for energy of a graph have been established in $[14,15]$ and it has found remarkable chemical applications in the molecular orbital theory of conjugated molecules $[6,10]$. Further studies on covering energy and dominating energy can be found in $[1,12]$.

\section{SEIDEL EnERGy}

Let $G$ be a simple graph of order $n$ with vertex set $V=\left\{v_{1}, v_{2}, \ldots, v_{n}\right\}$ and edge set $E$. The Seidel matrix of $G$ is the $n \times n$ matrix defined by $S(G):=\left(s_{i j}\right)$, where $s_{i j}= \begin{cases}-1 & \text { if } v_{i} v_{j} \in E \\ 1 & \text { if } v_{i} v_{j} \notin E \\ 0 & \text { if } v_{i}=v_{j}\end{cases}$

The characteristic polynomial of $S(G)$ is denoted by $f_{n}(G, \lambda)=\operatorname{det}(\lambda I-$ $S(G)$ ). The Seidel eigenvalues of the graph $G$ are the eigenvalues of $S(G)$. Since $S(G)$ is real and symmetric, its eigenvalues are real numbers. The Seidel energy [11] of $G$ is defined as $S E(G):=\sum_{i=1}^{n}\left|\lambda_{i}\right|$

2.1. Minimum Covering Seidel Energy. Let $G$ be a simple graph of order $n$ with vertex set $V=\left\{v_{1}, v_{2}, \ldots, v_{n}\right\}$ and edge set E. A subset $C$ of $V$ is called a covering set of $G$ if every edge of $G$ is incident to one vertex in $C$. Any covering set with minimum cardinality is called a minimum covering set. Let $C$ be a minimum covering set of a graph $G$. The minimum covering Seidel matrix of $G$ is the $n \times n$ matrix defined by $S_{C}(G):=\left(s_{i j}\right)$,

$$
\text { where } s_{i j}= \begin{cases}-1 & \text { if } v_{i} v_{j} \in E \\ 1 & \text { if } v_{i} v_{j} \notin E \\ 1 & \text { if } i=j \text { and } v_{i} \in C \\ 0 & \text { if } i=j \text { and } v_{i} \notin C\end{cases}
$$

The characteristic polynomial of $S_{C}(G)$ is denoted by $f_{n}(G, \lambda)=\operatorname{det}(\lambda I-$ $S_{C}(G)$ ). The minimum covering Seidel eigenvalues of the graph $G$ are the eigenvalues of $S_{C}(G)$. Since $S_{C}(G)$ is real and symmetric, its eigenvalues are real numbers and we label them in non-increasing order $\lambda_{1} \geqslant \lambda_{2} \geqslant \cdots \geqslant \lambda_{n}$. The minimum covering Seidel energy of $G$ is defined as $S E_{C}(G):=\sum_{i=1}^{n}\left|\lambda_{i}\right|$ Note that the trace of $S_{C}(G)=|C|$. 
Example 1: The possible minimum covering sets for the following graph $G$ [Figure 1] are i) $C_{1}=\left\{v_{1}, v_{2}, v_{5}\right\} \quad$ ii) $C_{2}=\left\{v_{2}, v_{4}, v_{5}\right\}$.

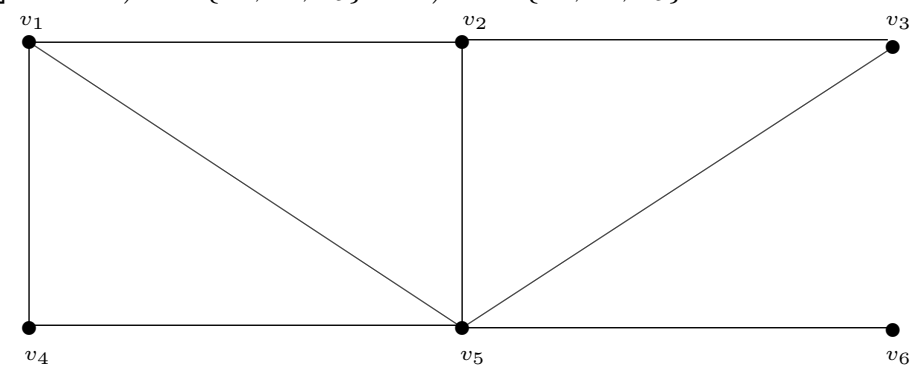

Figure - 1 Graph $G$

i) $S_{C_{1}}(G)=\left(\begin{array}{cccccc}1 & -1 & 1 & -1 & -1 & 1 \\ -1 & 1 & -1 & 1 & -1 & 1 \\ 1 & -1 & 0 & 1 & -1 & 1 \\ -1 & 1 & 1 & 0 & -1 & 1 \\ -1 & -1 & -1 & -1 & 1 & -1 \\ 1 & 1 & 1 & 1 & -1 & 0\end{array}\right)$

Characteristic equation is $\lambda^{6}-3 \lambda^{5}-12 \lambda^{4}+21 \lambda^{3}+49 \lambda^{2}-8 \lambda-12=0$.

Minimum covering Seidel eigenvalues are $\lambda_{1} \approx 0.537401577025226$, $\lambda_{2} \approx-0.472833908995256, \lambda_{3} \approx-2.000000000000006, \lambda_{4} \approx-2.000000000000006$, $\lambda_{5} \approx 3.000000000000075, \lambda_{6} \approx 3.935432331969967$.

Minimum covering Seidel energy, $S E_{C_{1}}(G) \approx 11.94566781799054$.

ii) $S_{C_{2}}(G)=\left(\begin{array}{cccccc}0 & -1 & 1 & -1 & -1 & 1 \\ -1 & 1 & -1 & 1 & -1 & 1 \\ 1 & -1 & 0 & 1 & -1 & 1 \\ -1 & 1 & 1 & 1 & -1 & 1 \\ -1 & -1 & -1 & -1 & 1 & -1 \\ 1 & 1 & 1 & 1 & -1 & 0\end{array}\right)$

Characteristic equation is $\lambda^{6}-3 \lambda^{5}-12 \lambda^{4}+21 \lambda^{3}+45 \lambda^{2}-16 \lambda-16=0$. Minimum covering Seidel eigenvalues are $\lambda_{1} \approx 0.720326520785057$, $\lambda_{2} \approx-0.50038699625876, \lambda_{3} \approx-1.824896034283096, \lambda_{4} \approx 2.707249041791971$, $\lambda_{5} \approx-2.195240160148165, \lambda_{6} \approx 4.092947628112992$

Minimum covering Seidel energy, $S E_{C_{2}}(G) \approx 12.04104638138004$.

$\therefore$ Minimum covering Seidel energy depends on the covering set.

\section{Minimum Covering Seidel Energy of Some Standard Graphs}

Theorem 3.1. For $n \geq 2$, the minimum covering Seidel energy of complete graph $K_{n}$ is $2(n-2)+\sqrt{n^{2}-2 n+5}$. 
Proof. $K_{n}$ is complete graph with vertex set $V=\left\{v_{1}, v_{2}, \ldots, v_{n}\right\}$. The minimum covering set is $C=\left\{v_{1}, v_{2}, v_{3}, \ldots \ldots, v_{n-1}\right\}$. Then

$$
S_{C}\left(K_{n}\right)=\left(\begin{array}{ccccccc}
1 & -1 & -1 & \ldots & -1 & -1 & -1 \\
-1 & 1 & -1 & \ldots & -1 & -1 & -1 \\
-1 & -1 & 1 & \ldots & -1 & -1 & -1 \\
\vdots & \vdots & \vdots & \ddots & \vdots & \vdots & \vdots \\
-1 & -1 & -1 & \ldots & 1 & -1 & -1 \\
-1 & -1 & -1 & \ldots & -1 & 1 & -1 \\
-1 & -1 & -1 & \ldots & -1 & -1 & 0
\end{array}\right)_{n \times n}
$$

Characteristic equation is $(-1)^{n}(\lambda-2)^{n-2}\left[\lambda^{2}+(n-3) \lambda-(n-1)\right]=0$.

Minimum covering Seidel $\operatorname{Spec}\left(K_{n}\right)$

$=\left(\begin{array}{ccc}2 & \frac{(n-3)+\sqrt{n^{2}-2 n+5}}{2} & \frac{(n-3)-\sqrt{n^{2}-2 n+5}}{2} \\ n-2 & 1 & 1\end{array}\right)$

Minimum covering Seidel energy is, $S E_{C}\left(K_{n}\right)$

$=|2|(n-2)+\left|\frac{(n-3)+\sqrt{n^{2}-2 n+5}}{2}\right|+\left|\frac{(n-3)-\sqrt{n^{2}-2 n+5}}{2}\right|$

$=2(n-2)+\sqrt{n^{2}-2 n+5}$.

Definition 3.2. The Cocktail party graph is denoted by $K_{n \times 2}$, is a graph having the vertex set $V=\bigcup_{i=1}^{n}\left\{u_{i}, v_{i}\right\}$ and the edge set $E=\left\{u_{i} u_{j}, v_{i} v_{j}: i \neq j\right\} \bigcup\left\{u_{i} v_{j}, v_{i} u_{j}\right.$ : $1 \leq i<j \leq n\}$.

Theorem 3.3. The minimum covering Seidel energy of cocktail party graph $K_{n \times 2}$, for $n \geq 2$ is $(4 n-7)+\sqrt{4 n^{2}-4 n+9}$. 
Proof. Let $K_{n \times 2}$ be the cocktail party graph with vertex set $V=\bigcup_{i=1}^{n}\left\{u_{i}, v_{i}\right\}$. The minimum covering set is $C=\bigcup_{i=1}^{n-1}\left\{u_{i}, v_{i}\right\}$. Then

$$
S_{C}\left(K_{n \times 2}\right)=\left(\begin{array}{c|ccccc|ccccc} 
& u_{1} & u_{2} & u_{3} & \ldots & u_{n} & v_{1} & v_{2} & v_{3} & \ldots & v_{n} \\
\hline u_{1} & 1 & -1 & -1 & \ldots & -1 & 1 & -1 & -1 & \ldots & -1 \\
u_{2} & -1 & 1 & -1 & \ldots & -1 & -1 & 1 & -1 & \ldots & -1 \\
u_{3} & -1 & -1 & 1 & \ldots & -1 & -1 & -1 & 1 & \ldots & -1 \\
\vdots & \vdots & \vdots & \vdots & & \vdots & \vdots & \vdots & \vdots & & \vdots \\
u_{n} & -1 & -1 & -1 & \ldots & 0 & -1 & -1 & -1 & \ldots & 1 \\
\hline v_{1} & 1 & -1 & -1 & \ldots & -1 & 1 & -1 & -1 & \ldots & -1 \\
v_{2} & -1 & 1 & -1 & \ldots & -1 & -1 & 1 & -1 & \ldots & -1 \\
v_{3} & -1 & -1 & 1 & \ldots & -1 & -1 & -1 & 1 & \ldots & -1 \\
\vdots & \vdots & \vdots & \vdots & & \vdots & \vdots & \vdots & \vdots & & \vdots \\
v_{n} & -1 & -1 & -1 & \ldots & 1 & -1 & -1 & -1 & \ldots & 0
\end{array}\right)
$$
$5)]=0$

Characteristic equation is $\quad \lambda^{(n-1)}(\lambda+1)(\lambda-4)^{(n-2)}\left[\left(\lambda^{2}+(2 n-7) \lambda-2(3 n-\right.\right.$

Minimum covering Seidel Spec $\left(K_{(n \times 2)}\right)$

$$
=\left(\begin{array}{ccccc}
0 & -1 & 4 & \frac{(2 n-7)+\sqrt{4 n^{2}-4 n+9}}{2} & \frac{(2 n-7)-\sqrt{4 n^{2}-4 n+9}}{2} \\
n-1 & 1 & n-2 & 1 & 1
\end{array}\right)
$$

Minimum covering Seidel energy, $S E_{C}\left(K_{n \times 2}\right)$

$=|0|(n-1)+|-1|(1)+|4|(n-2)+\left|\frac{(2 n-7)+\sqrt{4 n^{2}-4 n+9}}{2}\right|+\left|\frac{(2 n-7)-\sqrt{4 n^{2}-4 n+9}}{2}\right|$ $=(4 n-7)+\sqrt{4 n^{2}-4 n+9}$.

Theorem 3.4. For $n \geq 2$, the minimum covering Seidel energy of star graph $K_{1, n-1}$ is equal to $(n-2)+\sqrt{n^{2}-2 n+5}$.

Proof. Consider the star graph $K_{1, n-1}$ with vertex set $V=\left\{v_{0}, v_{1}, v_{2}, \ldots, v_{n-1}\right\}$. Minimum covering set is $C=\left\{v_{0}\right\}$. Then

$$
S_{C}\left(K_{1, n-1}\right)=\left(\begin{array}{ccccccc}
1 & -1 & -1 & \ldots & -1 & -1 & -1 \\
-1 & 0 & 1 & \ldots & 1 & 1 & 1 \\
-1 & 1 & 0 & \ldots & 1 & 1 & 1 \\
\vdots & \vdots & \vdots & \ddots & \vdots & \vdots & \vdots \\
-1 & 1 & 1 & \ldots & 0 & 1 & 1 \\
-1 & 1 & 1 & \ldots & 1 & 0 & 1 \\
-1 & 1 & 1 & \ldots & 1 & 1 & 0
\end{array}\right)_{n \times n}
$$


Characteristic equation is $(-1)^{n}(\lambda+1)^{n-2}\left[\lambda^{2}-(n-1) \lambda-1\right]=0$

Minimum covering Seidel Spec $\left(K_{1, n-1}\right)$

$=\left(\begin{array}{ccc}-1 & \frac{(n-1)+\sqrt{n^{2}-2 n+5}}{2} & \frac{(n-1)-\sqrt{n^{2}-2 n+5}}{2} \\ n-2 & 1 & 1\end{array}\right)$

Minimum covering Seidel energy is,

$$
\begin{aligned}
& S E_{C}\left(K_{1, n-1}\right)=|-1|(n-2)+\left|\frac{(n-1)+\sqrt{n^{2}-2 n+5}}{2}\right|+\left|\frac{(n-1)-\sqrt{n^{2}-2 n+5}}{2}\right| \\
& =(n-2)+\sqrt{n^{2}-2 n+5}
\end{aligned}
$$

Definition 3.5. The Crown graph $S_{n}^{0}$ for an integer $n \geq 2$ is the graph with vertex set $\left\{u_{1}, u_{2}, \ldots, u_{n}, v_{1}, v_{2}, \ldots, v_{n}\right\}$ and edge set $\left\{u_{i} v_{j}: 1 \leq i, j \leq n, i \neq j\right\}$. $\therefore S_{n}^{0}$ coincides with the complete bipartite graph $K_{n, n}$ with horizontal edges removed.

Theorem 3.6. For $n \geq 2$, the minimum covering Seidel energy of the crown graph $S_{n}^{0}$ is equal to $n(\sqrt{17}+2)-(\sqrt{17}+1)$.

Proof. For the crown graph $S_{n}^{0}$ with vertex set $V=\left\{u_{1}, u_{2}, \ldots, u_{n}, v_{1}, v_{2}, \ldots, v_{n}\right\}$ ,minimum covering set is $C=\left\{u_{1}, u_{2}, \ldots, u_{n}\right\}$.Then

$$
S_{C}\left(S_{n}^{0}\right)=\left(\begin{array}{c|ccccc|ccccc} 
& u_{1} & u_{2} & u_{3} & \ldots & u_{n} & v_{1} & v_{2} & v_{3} & \ldots & v_{n} \\
\hline u_{1} & 1 & 1 & 1 & \ldots & 1 & 1 & -1 & -1 & \ldots & -1 \\
u_{2} & 1 & 1 & 1 & \ldots & 1 & -1 & 1 & -1 & \ldots & -1 \\
u_{3} & 1 & 1 & 1 & \ldots & 1 & -1 & -1 & 1 & \ldots & -1 \\
\vdots & \vdots & \vdots & \vdots & & \vdots & \vdots & \vdots & \vdots & & \vdots \\
u_{n} & 1 & 1 & 1 & \ldots & 1 & -1 & -1 & -1 & \ldots & 1 \\
\hline v_{1} & 1 & -1 & -1 & \ldots & -1 & 0 & 1 & 1 & \ldots & 1 \\
v_{2} & -1 & 1 & -1 & \ldots & -1 & 1 & 0 & 1 & \ldots & 1 \\
v_{3} & -1 & -1 & 1 & \ldots & -1 & 1 & 1 & 0 & \ldots & 1 \\
\vdots & \vdots & \vdots & \vdots & & \vdots & \vdots & \vdots & \vdots & & \vdots \\
v_{n} & -1 & -1 & -1 & \ldots & -1 & 1 & 1 & 1 & \ldots & 0
\end{array}\right)_{(2 n \times 2 n)}
$$

Characteristic equation is $\left(\lambda^{2}+\lambda-4\right)^{n-1}\left[\lambda^{2}-(2 n-1) \lambda+(3 n-4)\right]=0$ Minimum covering $\operatorname{Seidel} \operatorname{Spec}\left(S_{n}^{0}\right)$ is

$$
\left(\begin{array}{cccc}
\frac{1+\sqrt{17}}{2} & \frac{1-\sqrt{17}}{2} & \frac{(2 n-1)+\sqrt{4 n^{2}-16 n+17}}{2} & \frac{(2 n-1)-\sqrt{4 n^{2}-16 n+17}}{2} \\
n-1 & n-1 & 1 & 1
\end{array}\right)
$$

Minimum covering Seidel energy $S E_{C}\left(S_{n}^{0}\right)$ is $\left|\frac{1+\sqrt{17}}{2}\right|(n-1)+\left|\frac{1-\sqrt{17}}{2}\right|(n-1)+\left|\frac{(2 n-1)+\sqrt{4 n^{2}-16 n+17}}{2}\right|+\left|\frac{(2 n-1)-\sqrt{4 n^{2}-16 n+17}}{2}\right|$ $=n(\sqrt{17}+2)-(\sqrt{17}+1)$. 
Theorem 3.7. The minimum covering Seidel energy of the complete bipartite graph $K_{m, n}$ is equal to $(n-1)+\sqrt{n^{2}+2(m-1) n+(m+1)^{2}}$.

Proof. For the complete bipartite graph $K_{m, n}(m \leq n)$ with vertex set $V=$ $\left\{u_{1}, u_{2}, \ldots, u_{m}, v_{1}, v_{2}, \ldots, v_{n}\right\}$, minimum covering set is $C=\left\{u_{1}, u_{2}, \ldots, u_{m}\right\}$ is a minimum covering set. Then

$S_{C}\left(K_{m, n}\right)=\left(\begin{array}{c|ccccc|ccccc} & u_{1} & u_{2} & u_{3} & \ldots & u_{m} & v_{1} & v_{2} & v_{3} & \ldots & v_{n} \\ \hline u_{1} & 1 & 1 & 1 & \ldots & 1 & -1 & -1 & -1 & \ldots & -1 \\ u_{2} & 1 & 1 & 1 & \ldots & 1 & -1 & -1 & -1 & \ldots & -1 \\ u_{3} & 1 & 1 & 1 & \ldots & 1 & -1 & -1 & -1 & \ldots & -1 \\ \vdots & \vdots & \vdots & \vdots & & \vdots & \vdots & \vdots & \vdots & & \vdots \\ u_{n} & 1 & 1 & 1 & \ldots & 1 & -1 & -1 & -1 & \ldots & -1 \\ \hline v_{1} & -1 & -1 & -1 & \ldots & -1 & 0 & 1 & 1 & \ldots & 1 \\ v_{2} & -1 & -1 & -1 & \ldots & -1 & 1 & 0 & 1 & \ldots & 1 \\ v_{3} & -1 & -1 & -1 & \ldots & -1 & 1 & 1 & 0 & \ldots & 1 \\ \vdots & \vdots & \vdots & \vdots & & \vdots & \vdots & \vdots & \vdots & & \vdots \\ v_{n} & -1 & -1 & -1 & \ldots & -1 & 1 & 1 & 1 & \ldots & 0\end{array}\right)_{(m+n) \times(m+n)}$

Characteristic equation is $(-1)^{m+n} \lambda^{m-1}(\lambda+1)^{n-1}\left[\lambda^{2}-(m+n-1) \lambda-m\right]=0$

Minimum covering $\operatorname{Seidel} \operatorname{Spec}\left(K_{m, n}\right)$ is

$\left(\begin{array}{cccc}0 & -1 & \frac{(m+n-1)+\sqrt{n^{2}+2(m-1) n+(m+1)^{2}}}{2} & \frac{(m+n-1)-\sqrt{n^{2}+2(m-1) n+(m+1)^{2}}}{2} \\ m-1 & n-1 & 1 & 1\end{array}\right)$

Minimum covering Seidel energy $S E_{C}\left(K_{m, n}\right)$ is

$|0|(m-1)+|-1|(n-1)+\left|\frac{(m+n-1)+\sqrt{n^{2}+2(m-1) n+(m+1)^{2}}}{2}\right|+$

$\left|\frac{(m+n-1)-\sqrt{n^{2}+2(m-1) n+(m+1)^{2}}}{2}\right|$

$=(n-1)+\sqrt{n^{2}+2(m-1) n+(m+1)^{2}}$.

\section{Properties of Minimum Covering Seidel Eigenvalues}

Theorem 4.1. Let $G$ be a simple graph with vertex set $V=\left\{v_{1}, v_{2}, \ldots, v_{n}\right\}$, edge set $E$ and $C=\left\{u_{1}, u_{2}, \ldots, u_{k}\right\}$ be a minimum covering set. If $\lambda_{1}, \lambda_{2}, \ldots, \lambda_{n}$ are the eigenvalues of minimum covering Seidel matrix $S_{C}(G)$ then (i) $\sum_{i=1}^{n} \lambda_{i}=|C|$.

(ii) $\sum_{i=1}^{n} \lambda_{i}^{2}=|C|+n^{2}-n$.

Proof. i) We know that the sum of the eigenvalues of $S_{C}(G)$ is the trace of $S_{C}(G)$

$\therefore \sum_{i=1}^{n} \lambda_{i}=\sum_{i=1}^{n} a_{i i}=|C|$. 
(ii) Similarly the sum of squares of the eigenvalues of $S_{C}(G)$ is trace of $\left[S_{C}(G)\right]^{2}$

$$
\begin{aligned}
\therefore \sum_{i=1}^{n} \lambda_{i}^{2} & =\sum_{i=1}^{n} \sum_{j=1}^{n} a_{i j} a_{j i} \\
& =\sum_{i=1}^{n}\left(a_{i i}\right)^{2}+\sum_{i \neq j} a_{i j} a_{j i} \\
& =\sum_{i=1}^{n}\left(a_{i i}\right)^{2}+2 \sum_{i<j}\left(a_{i j}\right)^{2} \\
& =|C|+2\left[m(-1)^{2}+\left(\frac{n^{2}-n}{2}-m\right)(1)^{2}\right] \\
& =|C|+n^{2}-n .
\end{aligned}
$$

\section{Bounds for Minimum Covering Seidel Energy}

Similar to McClelland's [15] bounds for energy of a graph, bounds for $S E_{C}(G)$ are given in the following theorem.

Theorem 5.1. Let $G$ be a simple graph with $n$ vertices and $m$ edges. If $C$ is the minimum covering set and $P=\left|\operatorname{det} S_{C}(G)\right|$ then

$\sqrt{\left(n^{2}-n+|C|\right)+n(n-1) P^{\frac{2}{n}}} \leq S E_{C}(G) \leq \sqrt{n\left(n^{2}-n+|C|\right)}$.

Proof.

Cauchy Schwarz inequality is $\left(\sum_{i=1}^{n} a_{i} b_{i}\right)^{2} \leq\left(\sum_{i=1}^{n} a_{i}^{2}\right)\left(\sum_{i=1}^{n} b_{i}^{2}\right)$

$$
\text { If } a_{i}=1, b_{i}=\left|\lambda_{i}\right| \text { then }\left(\sum_{i=1}^{n}\left|\lambda_{i}\right|\right)^{2} \leq\left(\sum_{i=1}^{n} 1\right)\left(\sum_{i=1}^{n} \lambda_{i}^{2}\right)
$$

By using Theorem 3.1 we have

$$
\begin{aligned}
{\left[S E_{C}(G)\right]^{2} } & \leq n\left(n^{2}-n+|C|\right) \\
\Longrightarrow S E_{C}(G) & \leq \sqrt{n\left(n^{2}-n+|C|\right)}
\end{aligned}
$$


Since arithmetic mean is not smaller than geometric mean we have

$$
\begin{aligned}
\frac{1}{n(n-1)} \sum_{i \neq j}\left|\lambda_{i} \| \lambda_{j}\right| & \geq\left[\prod_{i \neq j}\left|\lambda_{i}\right|\left|\lambda_{j}\right|\right] \frac{1}{n(n-1)} \\
& =\left[\prod_{i=1}^{n}\left|\lambda_{i}\right|^{2(n-1)}\right] \frac{1}{n(n-1)} \\
& =\left[\prod_{i=1}^{n}\left|\lambda_{i}\right|\right]^{\frac{2}{n}} \\
& =\left|\prod_{i=1}^{n} \lambda_{i}\right|^{\frac{2}{n}} \\
& =\left|\operatorname{det} S_{C}(G)\right|^{\frac{2}{n}}=P^{\frac{2}{n}} \\
\therefore \sum_{i \neq j}\left|\lambda_{i} \| \lambda_{j}\right| \geq n(n-1) P^{\frac{2}{n}} &
\end{aligned}
$$

Now consider, $\left[S E_{C}(G)\right]^{2}=\left(\sum_{i=1}^{n}\left|\lambda_{i}\right|\right)^{2}$

$$
\begin{aligned}
& =\sum_{i=1}^{n}\left|\lambda_{i}\right|^{2}+\sum_{i \neq j}\left|\lambda_{i}\right|\left|\lambda_{j}\right| \\
& \therefore \quad\left[S E_{C}(G)\right]^{2} \geq\left(|C|+n^{2}-n\right)+n(n-1) P^{\frac{2}{n}} \quad \text { [From (4.1)] } \\
& \text { i.e., } S E_{C}(G) \geq \sqrt{\left(|C|+n^{2}-n\right)+n(n-1) P^{\frac{2}{n}}}
\end{aligned}
$$

Theorem 5.2. If $\lambda_{1}(G)$ is the largest minimum covering Seidel eigen value of $S_{C}(G)$, then $\lambda_{1}(G) \geq \frac{n^{2}-n+|C|}{n}$.

Proof. Let $X$ be any nonzero vector. Then by [2], We have $\lambda_{1}(A)=\max _{X \neq 0}\left\{\frac{X^{\prime} A X}{X^{\prime} X}\right\}$ $\therefore \lambda_{1}(A) \geq \frac{J^{\prime} A J}{J^{\prime} J}=\frac{n^{2}-n+|C|}{n}$ where $J$ is a unit matrix $[1,1,1, \ldots, 1]^{\prime}$.

Similar to Koolen and Moulton's [13] upper bound for energy of a graph, upper bound for $S E_{C}(G)$ is given in the following theorem. 
Theorem 5.3. If $G$ is a graph with $n$ vertices and $m$ edges and $\left(n^{2}-n+|C|\right) \geq n$ then

$S E_{C}(G) \leq \frac{n^{2}-n+|C|}{n}+\sqrt{(n-1)\left[\left(n^{2}-n+|C|\right)-\left(\frac{n^{2}-n+|C|}{n}\right)^{2}\right]}$.

Proof.

Cauchy-Schwartz inequality is $\left[\sum_{i=2}^{n} a_{i} b_{i}\right]^{2} \leq\left(\sum_{i=2}^{n} a_{i}^{2}\right)\left(\sum_{i=2}^{n} b_{j}^{2}\right)$

$$
\text { Put } \begin{aligned}
a_{i}=1, b_{i}=\left|\lambda_{i}\right| \operatorname{then}\left(\sum_{i=2}^{n}\left|\lambda_{i}\right|\right)^{2} & =\sum_{i=2}^{n} 1 \sum_{i=2}^{n} \lambda_{i}^{2} \\
\Rightarrow\left[S E_{C}(G)-\lambda_{1}\right]^{2} & \leq(n-1)\left(n^{2}-n+|C|-\lambda_{1}^{2}\right) \\
\Rightarrow S E_{C}(G) & \leq \lambda_{1}+\sqrt{(n-1)\left(n^{2}-n+|C|-\lambda_{1}^{2}\right)}
\end{aligned}
$$

Let $f(x)=x+\sqrt{(n-1)\left(n^{2}-n+|C|-x^{2}\right)}$

$$
\text { For decreasing function } \begin{aligned}
f^{\prime}(x) \leq 0 & \Rightarrow 1-\frac{x(n-1)}{\sqrt{(n-1)\left(n^{2}-n+|C|-x^{2}\right)}} \leq 0 \\
& \Rightarrow x \geq \sqrt{\frac{n^{2}-n+|C|}{n}}
\end{aligned}
$$

Since $\left(n^{2}-n+|C|\right) \geq n$, we have $\sqrt{\frac{n^{2}-n+|C|}{n}} \leq \frac{n^{2}-n+|C|}{n} \leq \lambda_{1}$

$$
\begin{aligned}
& \therefore f\left(\lambda_{1}\right) \leq f\left(\frac{n^{2}-n+|C|}{n}\right) \\
& \text { i.e., } S E_{C}(G) \leq f\left(\lambda_{1}\right) \leq f\left(\frac{n^{2}-n+|C|}{n}\right) \\
& \text { i.e., } S E_{C}(G) \leq f\left(\frac{n^{2}-n+|C|}{n}\right) \\
& \text { i.e., } S E_{C}(G) \leq \frac{n^{2}-n+|C|}{n}+\sqrt{(n-1)\left[n^{2}-n+|C|-\left(\frac{n^{2}-n+|C|}{n}\right)^{2}\right]} .
\end{aligned}
$$

Recently Milovanović [16] et al. gave a sharper lower bounds for energy of a graph. In this paper similar bounds for minimum covering Seidel energy of a graph are established

Theorem 5.4. Let $G$ be a graph with $n$ vertices and $m$ edges. Let $\left|\lambda_{1}\right| \geq\left|\lambda_{2}\right| \geq$ $\ldots \geq\left|\lambda_{n}\right|$ be a non-increasing order of minimum covering Seidel eigenvalues of $S_{C}(G)$ and $C$ is minimum covering set then $S E_{C}(G) \geq \sqrt{n\left(n^{2}-n+|C|\right)-\alpha(n)\left(\left|\lambda_{1}\right|-\left|\lambda_{n}\right|\right)^{2}}$ where $\alpha(n)=n\left[\frac{n}{2}\right]\left(1-\frac{1}{n}\left[\frac{n}{2}\right]\right)$ and $[x]$ denotes the integral part of a real number 
Proof. Let $a, a_{1}, a_{2}, \ldots a_{n}, A$ and $b, b_{1}, b_{2}, \ldots b_{n}, B$ be real numbers such that $a \leq$ $a_{i} \leq A$ and $b \leq b_{i} \leq B \forall i=1,2, \ldots n$ then the following inequality is valid. $\left|n \sum_{i=1}^{n} a_{i} b_{i}-\sum_{i=1}^{n} a_{i} \sum_{i=1}^{n} b_{i}\right| \leq \alpha(n)(A-a)(B-b)$ where $\alpha(\mathrm{n})=n\left[\frac{n}{2}\right]\left(1-\frac{1}{n}\left[\frac{n}{2}\right]\right)$ and equality holds if and only if $a_{1}=a_{2}=\ldots=a_{n}$ and $b_{1}=b_{2}=\ldots=b_{n}$. If $a_{i}=\left|\lambda_{i}\right|, b_{i}=\left|\lambda_{i}\right|, a=b=\left|\lambda_{n}\right|$ and $A=B=\left|\lambda_{1}\right|$, then

$$
\left.\left|n \sum_{i=1}^{n}\right| \lambda_{i}\right|^{2}-\left(\sum_{i=1}^{n}\left|\lambda_{i}\right|\right)^{2} \mid \leq \alpha(n)\left(\left|\lambda_{1}\right|-\left|\lambda_{n}\right|\right)^{2}
$$

But $\sum_{i=1}^{n}\left|\lambda_{i}\right|^{2}=n^{2}-n+|C|$ and $S E_{C}(G) \leq \sqrt{n\left(n^{2}-n+|C|\right)}$ then the above inequality becomes

$$
\begin{gathered}
n\left(n^{2}-n+|C|\right)-\left(S E_{C}(G)\right)^{2} \leq \alpha(n)\left(\left|\lambda_{1}\right|-\left|\lambda_{n}\right|\right)^{2} \\
\text { i,e., } S E_{C}(G) \geq \sqrt{n\left(n^{2}-n+|C|\right)-\alpha(n)\left(\left|\lambda_{1}\right|-\left|\lambda_{n}\right|\right)^{2}}
\end{gathered}
$$

Theorem 5.5. Let $G$ be a graph with $n$ vertices and $m$ edges. Let $\left|\lambda_{1}\right| \geq\left|\lambda_{2}\right| \geq$ $\ldots \geq\left|\lambda_{n}\right|>0$ be a non-increasing order of minimum covering eigenvalues of $S_{C}(G)$ then $S E_{C}(G) \geq \frac{n^{2}-n+|C|+n\left|\lambda_{1}\right|\left|\lambda_{n}\right|}{\left(\left|\lambda_{1}\right|+\left|\lambda_{n}\right|\right)}$

Proof. Let $a_{i} \neq 0, b_{i}, r$ and $R$ be real numbers satisfying $r a_{i} \leq b_{i} \leq R a_{i}$, then the following inequality holds.[Theorem 2, [16]]

$$
\sum_{i=1}^{n} b_{i}^{2}+r R \sum_{i=1}^{n} a_{i} \leq(r+R) \sum_{i=1}^{n} a_{i} b_{i}
$$

Put $b_{i}=\left|\lambda_{i}\right|, a_{i}=1, r=\left|\lambda_{n}\right|$ and $R=\left|\lambda_{1}\right|$ then

$$
\begin{gathered}
\sum_{i=1}^{n}\left|\lambda_{i}\right|^{2}+\left|\lambda_{1}\right|\left|\lambda_{n}\right| \sum_{i=1}^{n} 1 \leq\left(\left|\lambda_{1}\right|+\left|\lambda_{n}\right|\right) \sum_{i=1}^{n}\left|\lambda_{i}\right| \\
\text { i.e., } n^{2}-n+|C|+\left|\lambda_{1}\right|\left|\lambda_{n}\right| n \leq\left(\left|\lambda_{1}\right|+\left|\lambda_{n}\right|\right) S E_{C}(G) \\
\therefore S E_{C}(G) \geq \frac{n^{2}-n+|C|+n\left|\lambda_{1}\right|\left|\lambda_{n}\right|}{\left(\left|\lambda_{1}\right|+\left|\lambda_{n}\right|\right)}
\end{gathered}
$$

Bapat and S.pati [3]proved that if the graph energy is a rational number then it is an even integer. Similar result for minimum covering Seidel energy is given in the following theorem. 
Theorem 5.6. Let $G$ be a graph with a minimum covering set $C$.If the minimum covering Seidel energy $S E_{C}(G)$ is a rational number, then $S E_{C}(G) \equiv|C|(\bmod 2)$.

Proof. The proof is similar to the theorem 5.4 of [12]

Acknowledgments: The authors thank the referees for their helpful comments and suggestions, which have improved the presentation of this paper. The First author is thankful to the University Grants Commission, Government of India for the financial support under the grant MRP(S)-0535/13-14/KAMY004/UGC-SWRO.

\section{REFERENCES}

[1] Adiga, C., Bayad, A., Gutman, I., and Srinivas, S.A., "The minimum covering energy of a graph", Kragujevac J. Sci., 34 (2012), 39 - 56.

[2] Bapat, R.B., Graphs and Matrices, page no. 32, Hindustan Book Agency, 2011.

[3] Bapat R.B., and Pati, S., "Energy of a graph is never an odd integer", Bull. Kerala Math. Assoc. 1 (2011), 129 - 132.

[4] Cvetković, D., and Gutman, I., (eds.), Applications of Graph Spectra, Mathematical Institution, Belgrade, 2009.

[5] Cvetković, D., and Gutman, I., (eds.), Selected Topics on Applications of Graph Spectra, Mathematical Institute, Belgrade, 2011.

[6] Graovac, A., Gutman, I., and Trinajstić, N., Topological Approach to the Chemistry of Conjugated Molecules, Springer, Berlin, 1977.

[7] Gutman, I., "The energy of a graph", Ber. Math-Statist. Sekt. Forschungsz.Graz, 103 (1978), $1-22$.

[8] Gutman, I., Li, X., and Zhang, J., Graph Energy,ed. by M.Dehmer, F.Emmert - Streib. Analysis of Complex Networks. From Biology to Linguistics, pp. 145 174, Wiley - VCH, Weinheim, 2009.

[9] Gutman, I., The energy of a graph: Old and New Results,ed.by A. Betten, A. Kohnert, R. Laue and A. Wassermann. Algebraic Combinatorics and Applications, pp. 196 - 211, Springer, Berlin, 2001.

[10] Gutman, I., and Polansky, O.E., Mathematical Concepts in Organic Chemistry, Springer, Berlin, 1986.

[11] Haemers, W.H., "Seidel Switching and Graph Energy", MATH Commun. Math. Comput. Chem, 68 (2012), 653-659.

[12] Kanna, M.R.R, Dharmendra, B.N., and Sridhara, G., "Minimum dominating energy of a graph", International Journal of Pure and Applied Mathematics, 85:4 (2013), 707-718.

[13] Koolen, J.H., and Moulton, V., "Maximal energy graphs", Adv.Appl. Math., 26 (2001), 47 52 .

[14] Liu, H. , Lu, M., and Tian, F., "Some upper bounds for the energy of graphs", Journal of Mathematical Chemistry, 41:1 (2007).

[15] McClelland, B.J., "Properties of the latent roots of a matrix: The estimation of $\pi$-electron energies", J. Chem. Phys., 54 (1971), 640 - 643.

[16] Milovanović, I.Ž., Milovanović, E.I., and Zakić, A., "A Short note on Graph Energy", MATH Commun. Math. Comput. Chem, 72 (2014), 179-182. 